\title{
NOTE ON SOME FRACTIONAL BI-INHOMOGENEOUS SCHRODINGER-CHOQUARD EQUATIONS
}

\author{
Lassaad Chergui ${ }^{1}$ \\ ${ }^{1}$ Qassim University
}

August 25, 2020

\begin{abstract}
In the subcritical energy case, local well-posedness is established in the radial energy space for a class of fractional inhomogeneous Choquard equations. The best constant of a Gagliardo-Nirenberg type inequality is obtained. Moreover, a sharp threshold of global existence versus blow-up dichotomy is obtained for mass super-critical and energy subcritical solutions.
\end{abstract}

\section{Hosted file}

inhomog choquard - version 2.pdf available at https://authorea.com/users/353666/articles/ 477460-note-on-some-fractional-bi-inhomogeneous-schrodinger-choquard-equations 